\title{
AFRODITA TODOPODEROSA: ENTRE EL ORFISMO Y LA MAGIA*
}

\author{
Emilio Suárez de la Torre \\ Universitat Pompeu Fabra \\ emilio.suarez@upf.edu
}

\section{RESUMEN}

Observaciones sobre el Papiro de Derveni, col. XXI, el Himno órfico 55 (1-14) y PGM IV (2898-2935 y 3207-3252), a propósito de los rasgos de la diosa que se destacan en cada uno de ellos, con una propuesta sobre las coincidencias.

PALABRAS ClaVE: Afrodita, poderes divinos, orfismo, magia.

ALMIGHTY APHRODITE: BETWEEN ORPHISM AND MAGIC

\section{ABSTRACT}

Remarks on Derveni Papyrus, col. XXI, Orphic Hymn 55 (1-14), and PGM IV (2898-2935 and 3207-3252), focused on the features of the goddess emphasized in each text, with a proposal concerning the coincidences.

KeYwords: Aphrodite, divine powers, Orphism, Magic.

Las creencias religiosas de los griegos de la Antigüedad presentan una combinación de elementos firmes y apenas modificados a lo largo del tiempo con otros que, diacrónica y sincrónicamente, se adaptaron a contextos y corrientes diversas. Si nos centramos en las figuras divinas, observamos esa combinación de perdurabilidad y flexibilidad de forma muy clara. No hay problema en asignar a cada una de ellas una función principal y unos poderes aceptados de forma unánime e inalterada, pero es notable el grado de variabilidad según los territorios y el período histórico al que se haga referencia. Si esta combinación de lo perdurable y lo variable es patente en el ámbito de la práctica religiosa y de los rituales, la flexibilidad, e incluso lo que podríamos llamar manipulación y apropiación de las figuras divinas aumenta considerablemente cuando se trata de corrientes religiosas al margen de las creencias y ritos 'oficiales' y cuando se suma a ello la especulación filosófico-teológica o de otra naturaleza.

En esta breve nota ejemplificaré lo que acabo de decir con cuatro testimonios, de épocas muy distintas, referentes a la diosa Afrodita. Si la sólida y fundamental 
monografía de Vinciane Pirenne-Delforge (1994) permite un enriquecedor paseo por el territorio griego centrado en los cultos y ritos de la diosa, con un análisis detallado de sus funciones, el posterior estudio de Gabriella Pironti (2007) incide precisamente en la necesidad de no considerar su perfil limitado al cliché de la "diosa del amor" y pone de manifiesto la importancia de su consideración paralela a la de Ares. No obstante, dentro de este rico panorama y de acuerdo con lo dicho más arriba, la utilización de la figura de Afrodita en los textos a que ahora haré referencia no se entiende sin la fundamental vinculación de la diosa a la pasión amorosa, sólo que proyectada en una dimensión cósmica, concebida de modo diferente en cada uno de ellos.

\section{(T1) P. DERVENI, COL. XXI}

La bibliografía sobre este comentario alegórico de una cosmogonía de Orfeo es inmensa ${ }^{2}$ y las tendencias interpretativas muy variadas, con discrepancias sobre numerosos detalles de cada una de sus 26 columnas. Como resumen de la estructura a reconstruir debe mencionarse la secuencia de deidades supremas (Noche, Urano, Crono, Zeus), con mitemas particulares no siempre coincidentes con los de otras cosmogonías y teogonías, aunque no tan lejos del esquema hesiódico como parece, al menos respecto a las deidades primordiales de la primera etapa ${ }^{3}$, aunque sí en la forma en que Zeus pasa a ser el motor de la configuración del universo en esta especie de nueva etapa creativa. En efecto, la divinidad fundamental para el origen de los seres será Zeus, concebido como principio y fin de todo ${ }^{4}$. La columna que traigo a colación nos da una muestra muy clara de la forma de trabajar del comentarista. En el relato general que parece reconstruirse, quien engendra a Afrodita es Zeus o, mejor dicho, la diosa surge a partir de la eyaculación creadora de Zeus 5 . La presencia de Afrodita

* El presente estudio se ha llevado a cabo en el marco del Proyecto de Investigación FFI201787558-P (AEI/FEDER, UE), financiado por el Ministerio de Ciencia, Economía y Productividad (MINECO).

${ }^{1}$ Sobre este aspecto véase Breitenberger (2007).

${ }^{2}$ Hasta 1997 se encuentra recogida en Funghi (1997). La posterior en Santamaría (2019a: 151-157).

${ }^{3}$ Cf. el cotejo de Santamaría (2019b).

${ }^{4}$ El proceso está muy bien definido en Bernabé (2002 y 2019).

${ }^{5}$ No está claro si habría un primer nacimiento de Afrodita a partir de los genitales de Urano y luego un segundo de la eyaculación de Zeus, tras engullir éste el pene de Urano, o si sólo hay que contar en el poema con la segunda alternativa. Por la primera se inclina Betegh (2004: 189), frente a la línea seguida por West (1983: 91), o Bernabé (loc. cit.). Este hecho no es significativo para mi argumentación, pero hay que tener en cuenta que aquí hay una referencia clara al nacimiento a partir de Zeus y no es tan clara la referencia al nacimiento a partir de Urano, a pesar del epíteto Urania. Lo que sí está claro es que esta designación fue aprovechada, como veremos, por el orfismo y la magia para profundizar en la valoración de Afrodita como deidad cósmica. 
era necesaria para sustentar la explicación del modo en que todos los entes y seres del universo van surgiendo una vez que entra en acción la diosa del amor y de la sexualidad, junto con su cortejo de diosas, personificadoras de aspectos del proceso amoro-

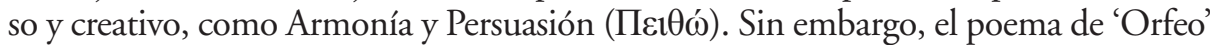
utilizaba un término que al comentarista le dio pie para una explicación cosmológica de compleja factura. Ese término es $\theta$ ópv $\eta l^{6}$, un hapax que es entendido (seguramente, con razón) como designación no exactamente del acto sexual, sino de la expulsión de materia seminal, es decir, la 'eyaculación' (como definición de la emisión seminal) o, en un sentido más cósmico, diseminación del esperma por el aire. Al aplicar su método alegórico y la especial orientación cosmológica de su método, el resultado es que el comentarista concibe como una unidad la multiplicidad de nombres que tienen que ver con las relaciones eróticas, y el propio Zeus ${ }^{7}$ acaba siendo una mera alternativa nominal en serie con Afrodita Urania, Armonía, Persuasión y los verbos

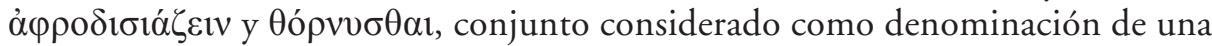
misma deidad ${ }^{8}$. Es decir, el surgimiento de todos los seres (según el comentarista)

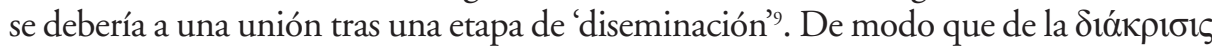
se pasa a la $\sigma 0 ́ \sigma \tau \alpha \sigma 1 \zeta$ mediante la $\mu \varepsilon \tilde{\xi} \xi 1 \zeta$. No obstante, a mi juicio, esta reinterpretación del comentarista, enmarcada dentro de su método la personal orientación de su método, no debe ocultar que, en el poema comentado, el papel de Afrodita tenía que quedar más destacado como diosa de la sexualidad y de la unión creadora, a pesar de que, sorprendentemente, nuestro autor parece derivar su nombre del verbo $\alpha \hat{\varphi} \varphi \rho o \delta$ -

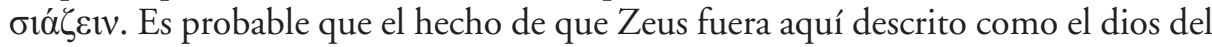
que dependía toda la actividad cósmica (y que impulsaría las acciones de ả $\varphi \rho o \delta$ -

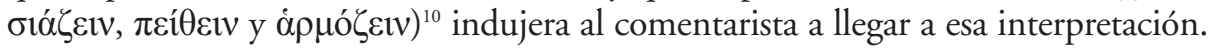

Sin embargo, lo que quiero subrayar es que, a través de la interpretación indicada, vislumbramos en el poema órfico un papel de Afrodita que, aunque marcadamente supeditado al de Zeus, supone una dimensión creadora universal que, a la vez que destaca la función sexual y engendradora de la diosa en el ámbito de la vida, tiene también una dimensión cósmica clave en la orientación del papel del Zeus omnipotente. Es decir, a pesar de las variaciones genealógicas del autor del poema y por encima de la interpretación del comentarista, no debemos olvidar que Afrodita recibe el epíteto de Urania y que, a partir de aquí, se convierte en una diosa imprescindible

${ }^{6}$ La discusión sobre este término (en cuanto a la lectura correcta) y su naturaleza (sustantivo o verbo) ha llenado muchas páginas. Remito de nuevo a Bernabé (2007), ad loc. He optado por aceptarlo como sustantivo, lo que sería un hapax, pero, en cualquier caso, no creo que haya duda de su relación semántica con las formas del radical $\theta o \rho v$ - que aparecen en el texto.

${ }^{7}$ Zeus había engullido el falo de Urano y su preñez lo convierte en "madre" de los dioses.

${ }^{8}$ Véase que, en contra de la larga tradición que ya vemos en Hesíodo, no es Eros el primer ente divino que marca la "sexualización" de la descendencia; cf. Bernabé (2007: 218), con referencias.

${ }^{9}$ Cf. Calame (1997: 70).

${ }^{10}$ Cf. Tortorelli-Ghidini (2019: 144), con referencia a Bernabé (2007). 
en la generación cósmica ${ }^{11}$. De hecho, lo cósmico y lo humano se complementan en esta visión, en la que hasta el propio comentarista hace de Afrodita un sinónimo del todopoderoso Zeus.

\section{(T2) H. ORPH. 55, A AFRODITA, LL. 1-14}

Paso ahora brevemente a un texto más tardío, correspondiente a la colección de himnos órficos, aunque de datación exacta discutida ${ }^{12}$. He seleccionado las primeras 14 líneas porque contienen algunos elementos que permiten una relación con el texto de Derveni y con el que luego veremos ${ }^{13}$. Desde luego el himno parte de una concepción de la diosa más tradicional en cuando a la genealogía, ya que aquí la denominación Urania apunta claramente al mito ya registrado en Hesíodo, como lo confirma la designación como "nacida en el mar" ( A simple vista podría dar la impresión de que la concepción del poder de la diosa

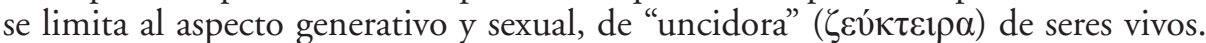
Sin embargo, véase que esta designación va seguida de una descripción de modalidades y ámbitos de acción muy destacable. El autor la califica de "madre de la necesidad" (ảvó $\gamma \kappa \eta)$, es decir, con el poder de controlar poco menos que el destino de los individuos. Esto se aclara con la expresión "pues todo procede de ti" y, además,

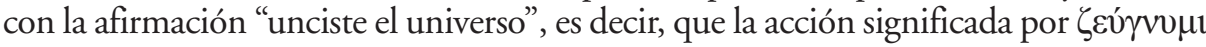

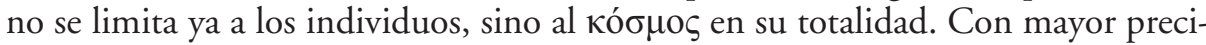
sión se añade que su ámbito de actuación es triple (porque ella "genera todo"): celestial, terrenal y submarino. Además, aparte de extenderse en la función de la diosa en cuanto a lo placentero (banquete, sexual y generativo (volverá a aparecer el verbo $\zeta \varepsilon v ́ \gamma v 0 \mu$ en 1. 13), se le asigna un rango muy elevado en relación con los demás dioses, tal como refleja la expresión $\theta \varepsilon \tilde{\omega} v \sigma \kappa \eta \pi \tau o \tilde{\chi} \chi \varepsilon^{14}(11)$.

Observamos, pues, en relación con el texto anterior, cierta línea de continuidad en la asignación a Afrodita de un poder que, sin apartarse del ámbito generativo, se extiende a todas las esferas posibles, del cosmos y de la vida. Tengamos en cuenta que ya en el texto de Derveni, con independencia de la opinión del comentarista, la tradición seguida en la argumentación refleja lo que podríamos llamar una "sexualización" de todo el proceso creativo que ensalza la figura de Afrodita en el conjunto del panteón griego.

${ }^{11}$ Cf. supra, n. 5.

${ }^{12}$ Oscila entre los siglos II y $\mathrm{V} \mathrm{d}$. C.

${ }^{13}$ El resto del himno se detiene sustancialmente en la enumeración alternativa de los lugares que constituyen las posibles moradas de la diosa, además de la petición final.

${ }^{14}$ Coincido con la editora (Ricciardelli, 2000, ad loc.) en la unión sintáctica de $\sigma \kappa \eta \pi \tau$ oṽ $\varepsilon$ con $\theta \varepsilon \tilde{\omega} v$ y no con el sustantivo que le precede ( $\sigma 0 ́ v \delta \alpha \iota \tau \imath$ ), que minusvaloraría el ensalzamiento de Afrodita. 
La plegaria que aquí se recoge forma parte de un hechizo de atracción o ảy pero su contenido presenta características muy especiales, debido a la adopción de elementos de origen iranio adaptados a la finalidad erótica mediante la inclusión de una historiola de difícil parangón y no exenta de problemas interpretativos ${ }^{15}$. La receta incluye las instrucciones de la praxis y dos invocaciones para la persuasión de Afrodita de muy distinto tono: una amenazante (11. 2898-2910), que ahora no comentaré, y otra elogiosa (1l. 2911-2935), en la que ahora me centro ${ }^{16}$. Esta parte no puede ser más trasparente en cuanto a la definitiva creación de un perfil de Afrodita como deidad superior con poderes cósmicos. El comienzo de la plegaria ya es de por sí muy expresivo. Desde luego, el nacimiento de Afrodita sigue el modelo hesiódico,

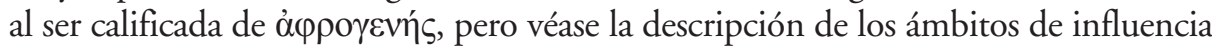
de la diosa: engendradora de dioses y hombres, celestial, subterránea, Naturaleza madre de todo, indominable, que unes unos seres con otros, que haces girar en derredor el gran fuego... Se plasma aquí de forma muy clara el dominio no sólo sobre dioses y hombres, sino también sobre los ámbitos celestes y terrenales, además de introducir una identifi-

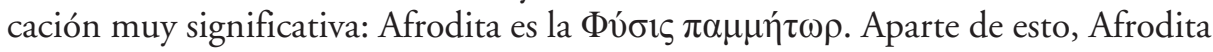
tiene el dominio del Sol, designado aquí con el nombre iranio Barza. Sin entrar ahora con detalle en la problemática que implican las líneas siguientes, en cuanto a los términos e identidad de Zov́ $\rho \omega$ y Pov́ ${ }^{9} \omega^{17}$, lo que queda claro es que de Afrodita dependen los movimientos del sol y estrellas, de las aguas y nada menos que del "ombligo del mundo"18. Además, el plano astral de la diosa se combina con el de su capacidad de unir a todos los seres, como se sintetiza en el adjetivo $\alpha \lambda \lambda \lambda \eta \lambda \sigma \tilde{\chi} \chi \varsigma^{19}$.

${ }^{15}$ Para los problemas planteados por esta receta de atracción, remito a mi comentario en Suárez de la Torre (2020), con análisis de detalle del texto. Ahora selecciono sólo los aspectos que permite una conexión con los textos comentados hasta ahora.

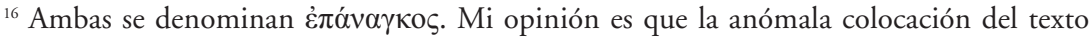
amenazante primero y del persuasivo después puede deberse a vicisitudes de la transmisión y manipulación del texto. De hecho la inversión de la secuencia conservada constituye un conjunto coherente perfectamente posible como ordenación original.

${ }^{17}$ Como indico en el estudio citado en n. 15, entiendo que hacen referencia a Afrodita. Véase la nota siguiente.

${ }^{18}$ Mi explicación de lo que aquí se describe es:

1. Afrodita hace girar a Barza.

2. Afrodita mantiene a Barza en continuo movimiento circular.

3. Afrodita lleva todo a cumplimiento (la mezcla del agua sagrada).

4. Afrodita mueve a una entidad divina no mencionada, ombligo del mundo, en el plano astral.

5. Afrodita, en paralelo, mueve a Hímeros dentro de las almas humanas.

${ }^{19}$ Sobre todos estos aspectos de Afrodita en este hechizo, véanse las páginas dedicadas al mismo

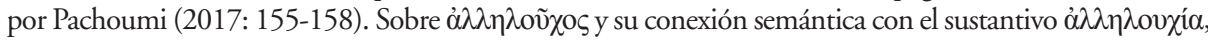
presente en Epicuro y los neoplatónicos, véase p. 156, con referencias en nn. 505-506. 
Se da el caso de que la identificación de Afrodita como Physis vuelve a aparecer en otra receta, en este caso adivinatoria. Se trata del $\varphi 1 \alpha \lambda o \mu \alpha v \tau \varepsilon i ̃ o v$ de $P G M$ IV (3207-3252). En él se presentan tres breves invocaciones a la diosa. Pues bien, en la tercera plegaria, Afrodita (que es también invocada con los nombres de Ilaouch

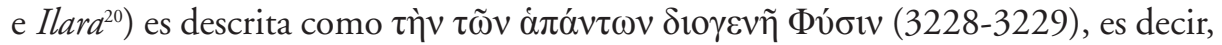
una nueva asimilación con la Naturaleza, con un calificativo que se presta a una doble interpretación si tenemos en cuenta un posible influjo órfico: Afrodita es la physis,

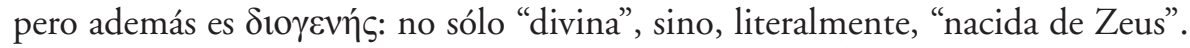

Por último, no olvidemos que, entre los Himnos Órficos, el número 10, dedicado a

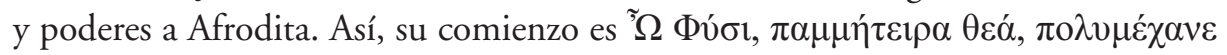

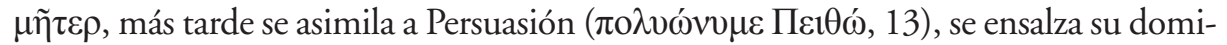

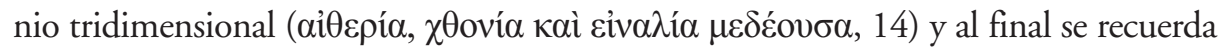

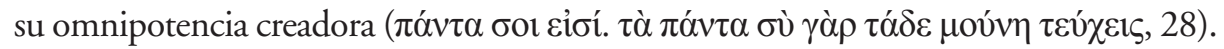

\section{CONCLUSIÓN}

Sumado todo ello, y aunque quizá no sea prudente hablar de influjos directos (en este caso) entre los textos órficos y los mágicos ${ }^{21}$, lo cierto es que se confirma cierta línea de continuidad en la construcción de una dimensión universal de Afrodita compartida por el orfismo y las recetas mágicas comentadas. La amplitud de conocimientos y recursos de aquellos que elaboraron las recetas mágicas, y muy especialmente la de quienes crearon y/o reunieron las del $P G M \mathrm{IV}$, está fuera de toda duda. Entre esos conocimientos está la de las tradiciones hímnicas de diversas culturas y, sobre todo, de la griega, como muestra un análisis de las coincidencias entre himnos órficos y mágicos, bien señaladas en los estudios recientes ${ }^{22}$. En este breve estudio sólo he pretendido poner de manifiesto la existencia de una concepción de la diosa Afrodita que, adaptada a los diferentes enfoques y creencias de los autores de los textos, se mantiene durante siglos y puede rastrearse en los textos seleccionados.

\footnotetext{
${ }^{20}$ Esta designación no tiene paralelo en los PGM.

${ }^{21}$ Sobre esta relación, indiscutible en muchos aspectos, véanse los fundamentales trabajos de Martín Hernández (2006, 2010).

${ }^{22}$ Petrovic (2015), Bortolani (2016), Herrero Valdés (2016) y Blanco Cesteros (2017).
} 


\section{TEXTOS COMENTADOS}

\section{Papiro de Derveni, col. $\mathrm{XXI}^{23}$}

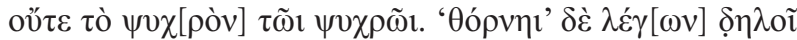

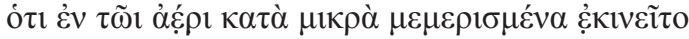

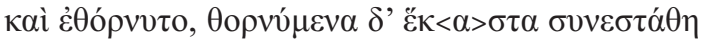

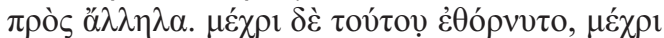

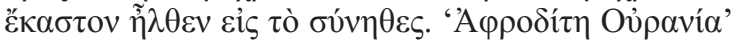

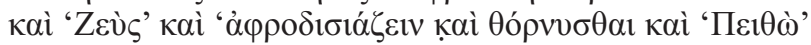

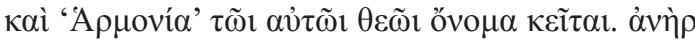

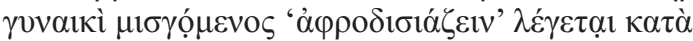

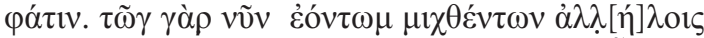

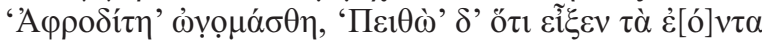

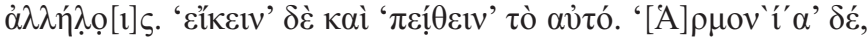

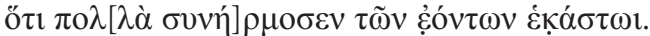

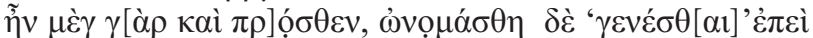

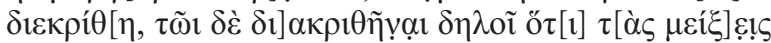

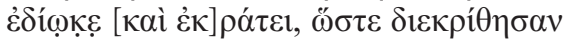

$$
\text { ]. [...]v. [ ]võv }
$$

Ni lo frío con lo frío. Al decir "por diseminación" deja claro que, dividido en pequeñas partículas, todo se movía y se diseminaba y, según se diseminaba, cada cosa llegó a una unión con otra. Y se fueron uniendo hasta el instante en que cada cosa llegó a su estado habitual. "Afrodita Urania" y "Zeus" y "hacer lo de Afrodita" y "diseminar" y "Persuasión" y "Armonía" es el nombre para una misma divinidad. Cuando un hombre se une a una mujer se suele decir que "hacen lo de Afrodita". Porque todos los seres que ahora están unidos entre sí recibieron el nombre de "Afrodita", y "Persuasión", porque los seres cedieron unos a otros, porque "ceder" y "obedecer" es lo mismo. Y "Armonía" porque se armonizaron entre sí muchos seres a cada uno de ellos. En efecto, ya existían antes, pero se denominó "llegar a ser" porque se separaron, pero el haberse separado pone de manifiesto que perseguían las uniones y dominaban, de modo que se separaron... ahora...

\section{H. Orph. 55, a Afrodita (1l. 1-14) $)^{24}$}

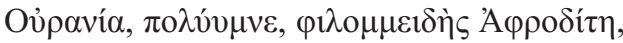

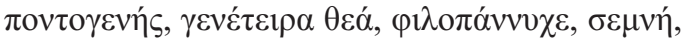

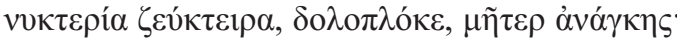

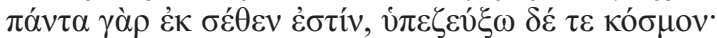

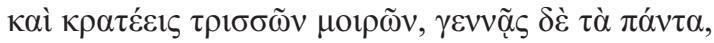

${ }^{23}$ Sigo la edición de Bernabé (2007).

${ }^{24}$ Sigo la edición de Ricciardelli (2000). 


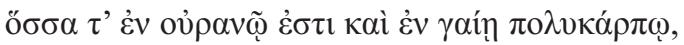

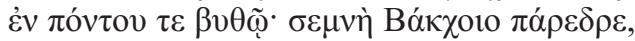

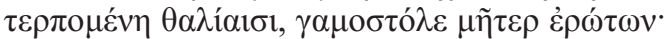

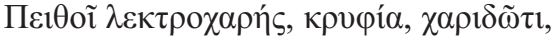

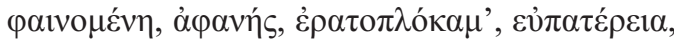

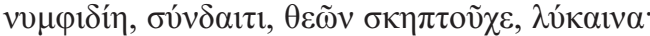

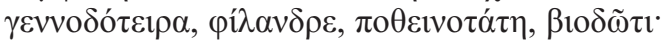

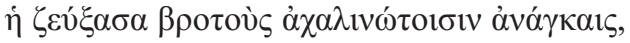

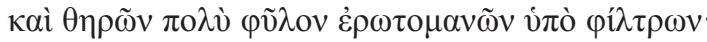

Urania, la muy cantada, Afrodita que ama la sonrisa, nacida en el ponto, diosa engendradora, amante de la nocturna fiesta, venerable, uncidora nocturna, trenzadora de engaños, madre de la Necesidad. Todo, sí, procede de ti y tú unciste el universo y tienes poder en los tres dominios y todo lo engendras, tanto lo que hay en el cielo y en la tierra fructífera como en la profundidad del mar; venerable colaboradora de Baco, que te deleitas con los banquetes, madre de los amores, que conciertas los matrimonios; Persuasión que haces gozar del lecho, furtiva, dadora de gracia, visible, invisible, de trenzas deseables, de noble padre, nupcial, comensal, portadora del cetro de los dioses, loba; dadora del linaje, amante de los hombres, la más deseada, dadora de vida; eres la que ha uncido a los mortales con necesidades irrefrenables y también a la numerosa raza de los animales, por obra de filtros que hacen enloquecer de pasión.

\section{3. $P G M$ IV 2911-2935}

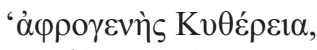

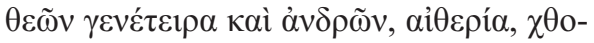

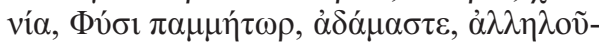

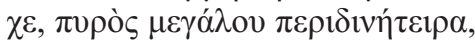

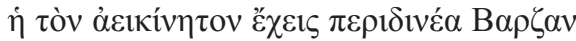

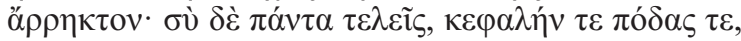

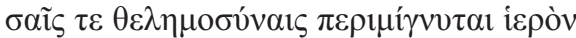

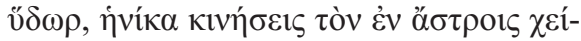

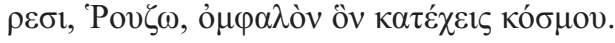

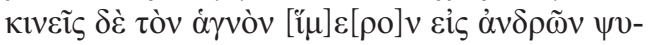

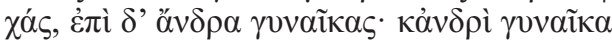

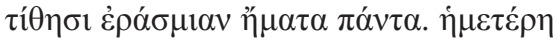

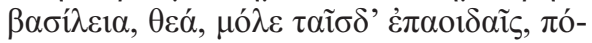

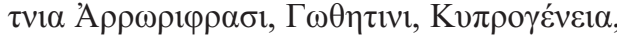

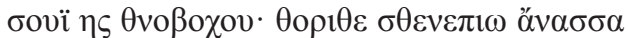

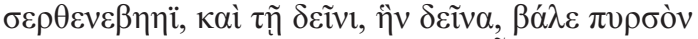

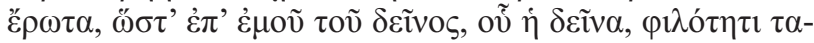

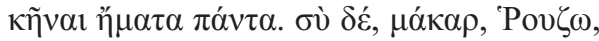

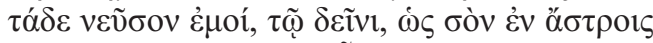

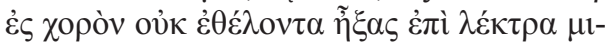

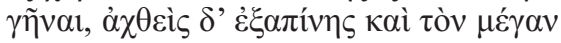

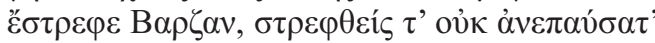

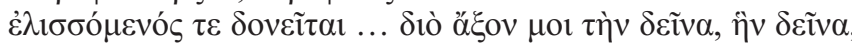

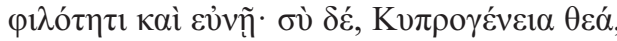

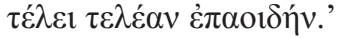


Citerea nacida de la espuma,

engendradora de dioses y hombres, celestial,

subterránea, Naturaleza madre de todo, indominable,

que unes unos seres con otros, que haces girar en derredor el gran fuego, que mantienes en eterno movimiento a Barza, que gira en derredor, indeleble; y tú todo lo llevas a la perfección, cabeza y pies,

y por tu voluntad se entremezcla el agua sagrada,

cuando tú, Ruzo, con tus manos mueves entre los astros

al que sustentas como ombligo del mundo.

Tú induces el puro deseo en las almas de los hombres,

y (atraes) a las mujeres a los hombres;

y eres la que hace a la mujer deseable todos los días.

Reina nuestra, diosa, acude a estos cantos, señora

Arrorifrasi, Gotetini, Ciprogenia

soui es tnobocu, torite, stenepio, soberana

sertenebeei, y a fulana, hija de mengana, arrójale ardiente

pasión, de modo que se consuma todos los días de amor

por mí, fulano, hijo de mengana. Y tú, bienaventurada, Zuro,

concédeme esto a mí, a fulano, igual que al coro que hay entre las estrellas llevaste al que no quería para unirse a ti en el lecho e hizo girar

al gran Barza, y éste, en cuanto comenzó a girar, no dejó de hacerlo

y en torbellino se agita... por ello, tráeme a fulana, hija de mengana,

al amor y al lecho; y tú, diosa en Chipre nacida,

lleva a término el encantamiento".

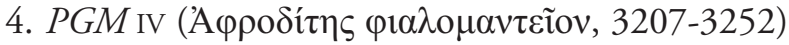

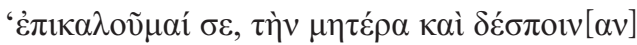

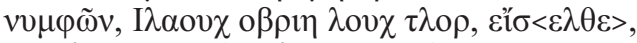

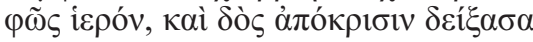

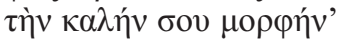

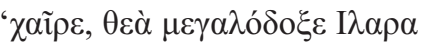

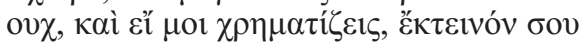

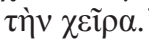

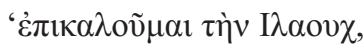

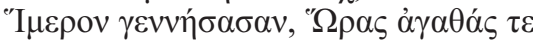

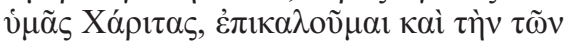

$\dot{\alpha} \pi \alpha ́ v \tau \omega v \delta 10 \gamma \varepsilon v \tilde{\eta} \Phi v ́ \sigma ı v, \delta i ́ \mu \rho \rho \varphi v, \dot{\alpha} \mu \varepsilon-$

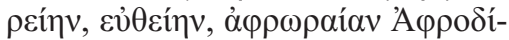

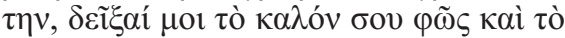

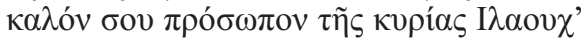

Te invoco, madre y señora de las Ninfas, Ilaouch obrie louch tlor, entra, luz sagrada, y dame una respuesta tras mostrarme tu hermosa forma... Salve, Ilara ouch, diosa gloriosísima, y si me vas a dar tu oráculo, extiende tu mano...Yo te invoco, Ilaouch, que engendraste al Deseo, las Horas y a vosotras, buenas Gracias, invoco también a la Naturaleza, de Zeus nacida, biforme, indivisible, recta, Afrodita la que tiene la belleza de la espuma, muéstrame tu hermosa luz y tu hermoso rostro de señora Ilaouch. 


\section{REFERENCIAS BIBLIOGRÁFICAS}

Bernabé, A. (2002): “La théogonie orphique du papyrus de Derveni”, Kernos 15: 91-129.

Bernabé, A. (2007): Poetae Epici Graeci. Testimonia et Fragmenta, Pars II, Orphicorum et Orphicis similium testimonia et fragmenta, fasc. 3 : Musaeus, Linus, Epimenides, Papyrus Derveni, Indices, Walter de Gruyter, Berolini-Novi Eboraci.

Bernabé, A. (2019): "The Commentary of the Derveni Papyrus: Pre-Socratic Cosmogonies at Work", en M. A. Santamaría (ed.), The Derveni Papyrus. Unearthing Ancient Mysteries, Brill, LeidenBoston, pp. 108-125.

Betegh, G. (2004): The Derveni Papyrus. Cosmology, Theology, and Interpretation, Cambridge University Press, Cambridge.

Blanco Cesteros, M. (2017): Edición y comentario de los himnos a Apolo, Helio y el dios supremo de los papiros mágicos griegos [Tesis doctoral inédita], Universidad de Valladolid.

Bortolani, L. M. (2016): Magical Hymns from Roman Egypt. A Study of Greek and Egyptian Traditions of Divinity, Cambridge University Press, Cambridge.

Breitenberger, B. (2007): Aphrodite and Eros. The Development of Erotic Mythology in Early Greek Poetry and Cult, Routledge, New York - London.

Calame, C. (1997): «Figures of Sexuality and Initiatory Transition in the Derveni Theogony and its Commentary», en A. LaKs - G. W. Most (eds.), Studies on the Derveni Papyrus, University Press, Oxford, pp. 65-80.

Funghi, M. S. (1997): «Bibliography of the Derveni Papyrus», en A. LaKS - G. W. Most (eds.), Studies on the Derveni Papyrus, University Press, Oxford, pp. 175-185.

HERRERO VALDÉs, F. (2016): Edición, traducción y comentario de los himnos mágicos griegos [Tesis doctoral inédita], Universidad de Málaga.

Kouremenos, Th. - Parássoglou, G. M. - Tsantsanoglou, K. (2006): The Derveni Papyrus (edited with introduction and commentary), Leo S. Olschki, Firenze.

LaKs, A. - Most, G. W. (eds.) (1997): Studies on the Derveni Papyrus, University Press, Oxford.

Martín Hernández, R. (2006): El orfismo y la magia [Tesis doctoral inédita], Universidad Complutense, Madrid.

Martín Hernández, R. (2010): Orfeo y los magos. La literatura órfica, la magia y los misterios, Abada Editores, Madrid.

Pachoumi. E. (2017): The Concept of the Divine in the Greek Magical Papyri, Mohr Siebeck, Tübingen.

Petrovic, I. (2015): "Hymns in the Papyri Graecae Magicae", in A. FAulKner - O. Hodkinson (eds.), Hymnic Narrative and the Narratology of Greek Hymns, Brill, Leiden - Boston, pp. 244-267.

Pirenne-Delforge, V. (1994): L'Aphrodite grecque, CIERGA, Athènes-Liège (Kernos suppl. 4).

Pironti, G. (2007): Entre Ciel et Guerre. Figures d'Aphrodite en Grèce ancienne, CIERGA, Liège (Kernos suppl. 18).

Ricciardelli, G. (2000): Inni Orfici, Fondazione Lorenzo Valla, Arnoldo Mondadori, Milano - Roma.

Santamaría, M. A. (ed.) (2019a): The Derveni Papyrus. Unearthing Ancient Mysteries, Brill, Leiden - Boston.

Santamaría, M. A. (2019b), «The Orphic Poem of the Derveni Papyrus an Hesiod's Theogony», en M. A. Santamaría (ed.), The Derveni Papyrus. Unearthing Ancient Mysteries, Brill, Leiden Boston, pp. 47-64. 
SuÁrez de la Torre, E. (2020), “Afrodita en los PGM”, en E. SuÁrez de la Torre - I. Canzobre MarTínez - C. SÁnCheZ-MAÑAS (eds.), Ablanathanalba. Magia, Cultura y sociedad en el Mundo Antiguo, Dykinson, Madrid, pp. 49-72.

SuÁrez de la Torre, E. - Canzobre Martínez, I. - SÁnchez-Mañas, C. (eds.) (2020): Ablanathanalba. Magia, Cultura y sociedad en el Mundo Antiguo, Dykinson, Madrid.

Tortorelli Ghidini, M. (2019): “Aphrodite Urania and Uranus Euphronides in the Derveni Papyrus: A Semantic Genealogy”, en M. A. Santamaría (ed.), The Derveni Papyrus. Unearthing Ancient Mysteries, Brill, Leiden-Boston, pp. 143-149.

West, M. L. (1983): The Orphic Poems, Clarendon Press, Oxford. 
